R. Zobel

Nagoya Math. J.

Vol. 78 (1980), 57-63

\title{
ZUM BEGRIFF DER BORELMESSBARKEIT
}

\author{
ROBERT ZOBEL
}

In dieser Arbeit werden die Voraussetzungen untersucht, die an einen topologischen Raum $(X, \subseteq)$ mit dem System offener Mengen $\subseteq$ zu stellen sind, damit einige der in der Literatur geläufigen Begriffe der Borelmeßbarkeit reellwertiger Funktionen äquivalent sind.

$\mathfrak{B}(\mathcal{S})$ sei die von $\subseteq$ erzeugte $\sigma$-Algebra. $\Re$ sei das System der kompakten Mengen von $(X, \subseteq)$. $\Re(\Re)$ sei der von $\Re$ erzeugte $\sigma$-Ring. $(X, \subseteq)$ ist genau dann $\sigma$-kompakt, d. h.: abzählbare Vereinigung kompakter Mengen, wenn $\Re(\Re) \supset \mathfrak{B}(\subseteq)$ gilt. Im allgemeinen enthält $\Re(\Re)$ nur die $\sigma$-beschränkten Teilmengen von $\mathfrak{B}\left(\subseteq_{)}\right)$, das sind die Mengen mit $\sigma$ kompakter Obermenge. Sei $K \in \mathfrak{R}$ und $\mathfrak{S}_{K}$ die Spurtopologie von $\subseteq$ auf $K, \Re_{K}(\Re)$ die Spur des $\sigma$-Ringes $\Re(\Re)$ und $\mathfrak{B}_{K}(\subseteq)$ die Spur der $\sigma$-Algebra $\mathfrak{B}\left(S_{)}\right)$auf K. Dann gilt $\mathfrak{B}\left(\overleftarrow{S}_{K}\right)=\mathfrak{B}_{K}\left(S_{)}\right) \subset \Re_{K}(\Re)$.

Gilt speziell in $(X, \subseteq)$, daß jede kompakte Menge abgeschlossen isteine Forderung, die schwächer als das Hausdorff'sche Trennungsaxiom $T_{2}$ und stärker als $T_{1}$ ist-so erhält man $\Re(\Re) \subset \mathfrak{B}(\subseteq)$. $\Re(\Re)$ besteht dann genau aus den $\sigma$-beschränkten Teilmengen von $X$. Für $K \in \Re$ gilt $\mathfrak{B}\left(\Im_{K}\right)=\mathfrak{B}_{K}\left(S_{)}\right)=\mathfrak{R}_{K}(\Re) . \quad\left(X, S_{)}\right)$ist $\sigma$-kompakt genau dann, wenn $\mathfrak{R}(\Re)$ $=\mathfrak{B}(\mathbb{S})$ gilt.

Sei $(X, \subseteq)$ ein beliebiger, topologischer Raum und $\Re$ ein $\sigma$-Ring über $X$ mit $\Re \subset \Re$. Dann heißt ein $\mathrm{Ma} \beta \mu$ auf $(X, \Re)$ ein Borelmaß, wenn $\mu(K)<\infty$ für $K \in \Re$ gilt. Es heißt von innen regulär, wenn für $A \in \Re$ gilt $\mu(A)=\sup \{\mu(K): K \in \mathfrak{\Re} \wedge K \subset A\}$.

Sei $\mu$ speziell ein von innen reguläres Borelmaß auf $(X, \Re(\Re))$ und $\Re_{\mu}(\Re)$ die Vervollständigung von $\Re(\Re)$ bezüglich $\mu$. Dann ist

$$
\mathfrak{B}_{\mu}(\Re)={ }_{\mathrm{D}\{}\left\{A: A \subset X \wedge \bigwedge_{K \in \Re} A \cap K \in \Re_{\mu}(\Re)\right\} .
$$

$\sigma$-Algebra über $X$ und $\mu$ besitzt genau eine Fortsetzung $\bar{\mu}$ zu einem von innen regulären Borelmaß über $\left(X, \mathfrak{B}_{\mu}(\Re)\right)$, die wesentliche Fortsetzung

Received June 16, 1978. 
$\bar{\mu}(A)={ }_{\mathrm{Df}} \sup \{\mu(K): K \in \Re \wedge K \subset A\}[2]$.

Der Maßraum $\left(X, \mathfrak{B}_{\mu}(\mathfrak{\Omega}), \bar{\mu}\right)$ ist vollständig, er enthält sogar alle Teilmengen lokaler Nullmengen, denn jede lokale Nullmenge ist Nullmenge. Der Maßraum ist also Caratheodory-vollständig.

Eine Abbildung $f$ heißt Borelfunktion von $X$ genau dann, wenn $f: X$ $\rightarrow \boldsymbol{R}$ und für alle reelen Zahlen $c$ gilt $[f<c]=\{x: x \in X \wedge f(x)<c\}$ $\in \mathfrak{B}_{\mu}(\Re)$. Genau dann ist $f$ eine Borelfunktion, wenn für jedes $K \in \Re$ ein $g: K \rightarrow \boldsymbol{R}$ existiert mit $[g<c] \in \mathfrak{B}\left(\mathfrak{S}_{K}\right)$ für $c \in \boldsymbol{R}$ und $f(x)=g(x)$ f. ü. für $x \in K$, wenn also für jede kompakte Teilmenge $K$ von $X$ die Einschränkung $\left.f\right|_{K}$ auf $K$ f. ü. gleich einer "Borelfunktion im üblichen Sinn" ist. Ist $f$ Borelfunktion und $g: X \rightarrow R$ mit $f=g$ f. ü. so ist auch $g$ Borelfunktion. $f$ ist genau dann "Borelfunktion im Sinne von Halmos", wenn $f$ Borelfunktion ist und außerhalb einer $\sigma$-kompakten Menge verschwindet. Der Zusammenhang dieses Begriffes der Borelfunktion und der "application mesurable" von Bourbaki [1] wird im folgenden untersucht:

Für einen topologischen $\operatorname{Raum}(X, \widetilde{S})$ sind die folgenden Eigenschaften gleichwertig $[3,6]$ :

(1) Zu $x, y \in X$ mit $x \neq y$ gibt es eine stetige Abbildung $f: X \rightarrow[0,1]$ mit $f(x)=0$ und $f(y)=1$.

(2) Es gibt eine stetige und injektive Abbildung $\phi$ von $X$ in $[0,1]^{k}$ für eine geeignete Kardinalzahl $k$.

(3) $(X, \subseteq)$ ist $T_{1}$-Raum und jede auf einer kompakten Menge definierte, stetige Abbildung ist stetig auf $X$ fortsetzbar.

Ein topologischer Raum, der eine der Bedingungen (1)-(3) erfüllt, ist Hausdorff-Raum und alle seine kompakten Mengen sind abgeschlossen. Genau die Topologien $\subseteq$ auf $X$, die Verfeinerung einer vollständig regulären Topologie $\mathfrak{I} \subset \mathbb{S}$ sind, erfüllen (1)-(3). Die Eigenschaften sind von der Regularität des Raumes unabhängig und sind schwächer als die vollständige Regularität und erst recht die Normalität. Sie sind für Produkträume (Summenräume) genau dann erfüllt, wenn sie für jeden Koordinatenraum (Summanden) gelten. Sie sind hereditär.

SATZ 1. (X, S) sei ein lokal-kompakter, o-kompakter Hausdorff-Raum und $\mu$ ein von innen reguläres Borelma $\beta$ über $(X, \Re(\Re))$. Dann sind für eine Funktion $f: X \rightarrow R$ die folgenden Aussagen gleichwertig:

(1a) $f$ ist Borelfunktion.

(1b) $\quad Z u$ jedem $B \in \mathfrak{B}_{\mu}(\Re)=\mathfrak{R}_{\mu}(\Re)$ mit $\mu(B)<\infty$ und jedem $\varepsilon>0$ existiert 
eine kompakte Menge $K \subset B$ mit $\bar{\mu}(B-K)<\varepsilon$ und eine stetige Abbildung $f^{\prime}: X \rightarrow \boldsymbol{R}$ mit kompaktem Träger und $f^{\prime}(x)=f(x)$ für $x \in K$.

(1c) $f$ ist f. ̈̈. punktweiser Grenzwert einer Folge $\left(f_{n}\right)_{n=1}^{\infty}$ von stetigen Funktionen mit kompaktem Träger. (Dabei kann $\left(f_{n}\right)_{n=1}^{\infty}$ so gewählt werden, $d a \beta\left(f_{n}(x)\right)_{n=1}^{\infty}$ für alle $x \in X$ ab einem gewissen Index konstant ist.)

(1d) Es existiert eine Zerlegung von $X$ in eine abzählbare Folge $\left(K_{n}\right)_{n=1}^{\infty}$ disjunkter, kompakter Mengen und in eine $\mu$-Nullmenge $N \in \Re(\Re)$, derart, daß die Restriktion von $f$ auf jede der Mengen $K_{n}$ stetig ist.

Zum Beweis der Äquivalenzen $(1 \mathrm{a}) \leftrightarrow(1 \mathrm{~b}) \leftrightarrow(1 \mathrm{~d})$ sei auf die Literatur verwiesen [1,2]. Man beachte, daß ein lokal-kompakter Hausdorff-Raum vollständig regulär ist und damit die äquivalenten Bedingungen (1)-(3) erfüllt. Für einen $\sigma$-kompakten Hausdorff-Raum gilt darüber hinaus $\mathfrak{B}(\widetilde{S})=\mathfrak{R}(\Re) . \quad(1 \mathrm{c}) \rightarrow(1 \mathrm{a})$ gilt auch ohne Zusatzbemerkung. Zu zeigen bleibt $(1 \mathrm{~b}) \rightarrow(1 \mathrm{c})$ :

Es gibt eine Kette kompakter Mengen $\left(K_{n}\right)_{n=1}^{\infty}$, so daß jeder Punkt $x$ $\epsilon K_{n}$ innerer Punkt von $K_{n+1}$ ist und $X=\bigcup_{n=1}^{\infty} K_{n}$ gilt. Wegen (1b) gibt es eine kompakte Menge $L_{n} \subset K_{n}$ mit $\mu\left(K_{n}-L_{n}\right)<2^{-n}$ und eine stetige Abbildung $f_{n}^{\prime}: X \rightarrow \boldsymbol{R}$, die auf $L_{n}$ mit $f$ übereinstimmt. Weiter gibt es wegen des Urysohn-Lemmas eine stetige Abbildung $g_{n}: X \rightarrow \boldsymbol{R}$ mit kompaktem Träger und $g_{n}(x)=1$ für $x \in L_{n}$. Mit $f_{n}=f_{n}^{\prime} \cdot g_{n}$ erhält man die gesuchte Folge. Denn ist $x \in L=\liminf _{n \rightarrow \infty} L_{n}$, so gilt $f_{n}(x)=f(x)$ ab einem gewissen Index. Weiter ist $M=X-L \mu$-Nullmenge. Denn wenn eine kompakte Menge $K \subset M$ mit $\mu(K)>0$ existiert, so gilt wegen $K \subset$ $\bigcup_{n=1}^{\infty} \operatorname{Kern}\left(K_{n}\right)=X$ ab einem gewissen Index $n^{\prime}$ für alle $n \geqslant n^{\prime} K \subset$ $\operatorname{Kern}\left(K_{n}\right)$. Sei $s$ eine natürliche Zahl mit $2^{-s+1}<\mu(K)$ und $s \geqslant n^{\prime}$. Nun gilt $K \cap \cap_{n=s}^{\infty} L_{n}=\emptyset$. Wegen $K \subset K_{n}$ für $n \geqslant s$ folgt:

$$
\begin{aligned}
\mu\left(K \cap \bigcap_{n=s}^{\infty} L_{n}\right) & =\mu\left(K-\bigcup_{n=s}^{\infty}\left(K-L_{n}\right)\right) \\
& \geqslant \mu(K)-\sum_{n=s}^{\infty} \mu\left(K_{n}-L_{n}\right) \\
& \geqslant \mu(K)-\sum_{n=s}^{\infty} 2^{-n}=\mu(K)-2^{-s+1}>0 .
\end{aligned}
$$

Dies ist ein Widerspruch zu $K \cap \bigcap_{n=s}^{\infty} L_{n}=\emptyset$. Das folgende Lemma beschäftigt sich mit der Frage der Fortsetzbarkeit eines von innen 
regulären Borelmaßes, das auf einer Teilmenge eines topologischen Raumes gegeben ist, wenn die auf der Teilmenge gegebene Topologie feiner ist als die Spurtopologie:

Lemma. Seien (U, S), $(X, \mathfrak{I})$ Hausdorff-Räume mit $U \subset X$ und für den Unterraum $\left(U, \mathfrak{I}_{U}\right)$ von $(X, \mathfrak{I})$ gelte $\mathfrak{I}_{U} \subset \mathcal{S}$. Sei $\mathfrak{R}$ das System der kompakten Mengen von $(U, \mathcal{S})$ und $\mathfrak{I}$ das von $(X, \mathfrak{I})$. Dann besitzt jedes von innen reguläre Borelma $\beta \mu$ auf $(U, \Re(\Re))$ eine $\sigma$-superadditive Fortsetzung $\mu^{\prime}$ auf $(X, \Re(\Omega))$ in

$$
\mu^{\prime}(A)={ }_{\mathrm{Df}} \sup \{\mu(K): K \subset A \cap U \wedge K \in \Re\}
$$

für $\emptyset \neq A \in \mathfrak{R}(\Omega)$ und $\mu^{\prime}(\emptyset)=0$.

Ist $U$ o-kompact, so gilt $\Re(\Re)=\{A \cap U: A \in \Re(\Omega)\}$, und $\mu^{\prime}$ ist ein Maß; ist $\mu$ zusätzlich endlich, so ist $\mu^{\prime}$ von innen reguläres Borelmaß.

Beweis. Ist $K$ ein kompakter Unterraum von $(U, \mathfrak{S})$, also $\left(K, \widetilde{S}_{K}\right)$ kompakter Hausdorff-Raum, so ist erst recht $\left(K, \mathfrak{I}_{K}\right)$ wegen $\mathfrak{I}_{K} \subset \mathfrak{S}_{K}$ ein kompakter Hausdorff-Raum. Dann gilt aber bereits $\mathfrak{S}_{K}=\mathfrak{I}_{K}$. Es folgt $\Re \subset \mathfrak{L}$ und damit $\Re(\Re) \subset \Re(\mathfrak{R})$. Da $\mu$ von innen regulär ist, ist $\mu^{\prime}$ Fortsetzung von $\mu$. $\mu^{\prime}$ ist monoton und positiv. Sei $\left\{A_{i}: i=1,2, \cdots\right\} \subset$ $\Re(\mathfrak{R})$ ein disjunktes Mengensystem und $C_{i} \subset A_{i} \cap U$ kompakt mit $\mu\left(C_{i}\right)$ $+\varepsilon \cdot 2^{-i} \geqslant \mu^{\prime}\left(A_{i}\right)$ :

$$
\begin{aligned}
\sum_{i=1}^{\infty} \mu^{\prime}\left(A_{i}\right) & \leqslant \sum_{i=1}^{\infty}\left(\mu\left(C_{i}\right)+\varepsilon \cdot 2^{-i}\right)=\mu\left(\sum_{i=1}^{\infty} C_{i}\right)+\varepsilon \\
& \leqslant \mu^{\prime}\left(\sum_{i=1}^{\infty} A_{i}\right)+\varepsilon .
\end{aligned}
$$

Dies liefert die $\sigma$-Superadditivität. Ist $U \sigma$-kompakt, so gilt $U=\bigcup_{i=1}^{\infty} K_{i}$ mit $K_{i} \in \Re \subset \mathfrak{R}$, also $U \in \Re(\Re) \subset \Re(\Omega)$. Sei $K \in \mathfrak{R}$, so folgt $K \cap U=$ $\cup_{i=1}^{\infty} K \cap K_{i}$. Wegen $K \cap K_{i} \in \Re$ erhält man $K \cap U \in \Re(\Re)$ und $\Re \subset\{K$ $\cap U: K \in \mathbb{R}\} \subset \Re(\Re)$, also $\Re(\Re) \subset\{A \cap U: A \in \Re(\Omega)\} \subset \Re(\Re)$. Dies liefert

$$
\mu^{\prime}\left(\sum_{i=1}^{\infty} A_{i}\right)=\mu\left(\sum_{i=1}^{\infty} A_{i} \cap U\right)=\sum_{i=1}^{\infty} \mu\left(A_{i} \cap U\right)=\sum_{i=1}^{\infty} \mu^{\prime}\left(A_{i}\right)
$$

Ist $\mu$ endlich, so ist $\mu$ sogar beschränkt und alle kompakten Teilmengen von $(X, \mathfrak{I})$ besitzen daher endliches Maß. Nach Konstruktion ist mit $\mu$ auch $\mu^{\prime}$ von innen regulär.

SATz 2. (X, I) sei ein topologischer Raum, der eine der äquivalenten 
Trennungseigenschaften (1), (2) oder (3) erfülle, $\mu$ sei ein von innen reguläres Borelmaß über $\left(X, \mathfrak{B}_{\mu}(\Re)\right)$ und $f: X \rightarrow \boldsymbol{R}$.

Die folgenden Eigenschaften sind gleichwertig:

(2a) $f$ ist Borelfunktion.

(2b) $Z u$ jedem $B \in \mathfrak{B}_{\mu}(\Re)$ mit $\mu(B)<\infty$ und jedem $\varepsilon>0$ existiert eine kompakte Menge $K \subset B$ und eine stetige Abbildung $f^{\prime}: X \rightarrow \boldsymbol{R}$ mit $\mu(B-K)<\varepsilon$ und $f(x)=f^{\prime}(x)$ für $x \in K$.

(2c) Zu jedem $B \in \Re(\Re)$ mit $\mu(B)<\infty$ existiert eine Folge $\left(f_{n}\right)_{n=1}^{\infty}$ von stetigen Abbildungen $f_{n}: X \rightarrow \boldsymbol{R}$, so $d a \beta f(x)=\lim f_{n}(x) \mu-f$. ü. für $x \in B$ gilt.

(2d) $Z u$ jedem $B \in \Re(\Re)$ existiert eine Zerlegung von $B$ in eine höchstens abzählbare Folge $\left(K_{n}\right)_{n=1}^{\infty}$ kompakter Mengen und in eine $\mu$-Nullmenge $N \in \Re(\Re)$, derart, daß die Einschränkung von $f$ auf jede der Mengen $K_{n}$ stetig ist.

Beweis. Nach Voraussetzung ist $(X, \mathfrak{I})$ stetig und injektiv einbettbar in $([0,1], \subseteq)^{k}$, wobei $\subseteq$ die gewöhnliche Topologie des $[0,1]$ und $k$ eine geeignete Kardinalzahl ist. O.B.d.A. setzen wir $X \subset[0,1]^{k}$ voraus. Sei $B \in \Re(\Re)$ und $\mu(B)<\infty$. Dann gibt es eine $\sigma$-kompakte Menge $C$ mit $\mu(B-C)=0$. Die Einschränkung von $\mu$ auf den von den kompakten Teilmengen von $C$ erzeugten Ring $\Re_{C}$ kann nach dem Lemma zu einem von innen regulären Borelmaß $\mu_{c}$ auf den $\sigma$-Ring $\Re$, der von den kompakten Teilmengen von $[0,1]^{k}$ erzeugt wird, fortgesetzt werden. Es gilt dann

$$
\mu_{C}\left([0,1]^{k}\right)=\mu_{C}(C)=\mu(C)=\mu(B)=\mu_{C}(B) .
$$

Damit ist dann Satz 2 eine Folgerung aus Satz 1, denn $\left([0,1]^{k}, \Re, \mu_{C}\right)$ erfüllt die Voraussetzungen des Satzes 1 , wobei statt $f: X \rightarrow \boldsymbol{R}$ nun $f_{c}:[0,1]^{k} \rightarrow R$ mit $f_{c}(x)=f(x)$ für $x \in C$ und $f_{C}(x)=0$ für $x \notin C$ zu betrachten ist. Satz 2 ist unter schwächeren Voraussetzungen als den angegebenen nicht $\mathrm{zu}$ beweisen, denn:

Bemerkung. Genau dann gilt für jedes von innen reguläre Borelmaß eines topologischen Raumes $(X, \mathfrak{I}) \quad(2 \mathrm{a}) \leftrightarrow(2 \mathrm{~b})$, wenn $(X, \mathfrak{I})$ eine der äquivalenten Eigenschaften (1), (2) oder (3) erfüllt.

Beweis. Es ist zu zeigen, daß aus $(2 a) \leftrightarrow(2 b)$ für jedes von innen reguläre Borelmaß (1) folgt: Sei $\mu_{a}$ für $a \in X$ gegeben durch $\mu_{a}(M)=0$ 
für $a \notin M$ und $\mu_{a}(M)=1$ für $a \in M$. Sei $x, y \in X$ und $x \neq y . \quad \mu=\mu_{x}+\mu_{y}$ ist von innen reguläres Borelmaß auf $(X, \mathfrak{I})$. Durch $f: X \rightarrow \boldsymbol{R}$ mit $f(x)$ $=1$ und $f(z)=0$ für $z \neq x$ erhält man eine Borelfunktion auf $X$. Mithin gibt es eine stetige Abbildung, die $x, y$ trennt.

Analoge Betrachtungen gelten für $(2 \mathrm{a}) \leftrightarrow(2 \mathrm{c})$ : Ist $(X, \mathfrak{I})$ ein topologischer Raum, in dem jede kompakte Menge abgeschlossen ist, so gilt $(2 \mathrm{a}) \leftrightarrow(2 \mathrm{~d})$. (2a) ist dann bereits äquivalent zu

$\left(2 b^{*}\right) \quad \mathrm{Zu}$ jedem $B \in \mathfrak{B}_{\mu}(\Re)$ mit $\mu(B)<\infty$ und $\varepsilon>0$ existiert eine kompakte Menge $K \subset B$ mit $\mu(B-K)<\varepsilon$ und $\left.f\right|_{K}$ ist stetig.

Wie die abzählbaren, trivial topologisierten Räume zeigen, braucht ein topologischer Raum, der für jedes von innen reguläre Borelmaß $(2 a) \leftrightarrow(2 d)$ erfüllt, nicht einmal $T_{1}$ zu erfüllen. Sei nun $(X, \mathfrak{T})$ bis auf eine Nullmenge $\sigma$-kompakt. Gelten dann die Voraussetzungen des Satzes 2, so ist jede Borelfunktion $\mu$-f. ü.-Grenzwert von stetigen Funktionen. Es ist dann also $\mathfrak{B}_{\mu}(\Re)$ Vervollständigung der Baire'schen $\sigma$-Algebra, d. h. der kleinsten $\sigma$-Algebra, bzgl. der alle stetigen Funktionen meßbar sind. Erst recht gilt dann $\mathfrak{B}_{\mu}(\mathfrak{\Re})=\mathfrak{B}_{\mu}(\mathfrak{I})$, wobei $\mathfrak{B}_{\mu}(\mathfrak{I})$ die Vervollständigung von $\mathfrak{B}(\mathfrak{I})$ bzgl. $\mu$ ist.

Bemerkung. $(X, \mathfrak{T})$ sei ein topologischer Raum, $\mu$ ein von innen reguläres Borelmaß auf $\mathfrak{B}(\mathfrak{T}) . \mathfrak{B}_{\mu}^{*}(\mathfrak{I})$ sei die Caratheodory-Vervollständigung von $\mathfrak{B}(\mathfrak{I})$ um lokale Nullmengen. $\bar{\mu}$ sei die wesentliche Fortsetzung von $\mu$ auf $\mathfrak{B}_{\mu}^{*}(\mathfrak{I}) \subset \mathfrak{B}_{\mu}(\mathfrak{R})$. Ist dann in $\left(X, \mathfrak{B}_{\mu}^{*}(\mathfrak{I}), \bar{\mu}\right)$ jedes $\bar{\mu}$-absolut stetige Maß nach $\bar{\mu}$ differenzierbar, so gilt $\mathfrak{B}_{\mu}^{*}(\mathfrak{I})=\mathfrak{B}_{\mu}(\Re)$.

Beweis. In $\left(X, \mathfrak{B}_{\mu}^{*}(\mathfrak{T}), \bar{\mu}\right)$ gilt das Segal'sche Lokalisationsprinzip [4, 5]. Ist $A \in \mathfrak{B}_{\mu}(\Re)$, so ist

$$
\mathfrak{i}=\left\{B: B \in \mathfrak{B}_{\mu}^{*}(\mathfrak{I}) \wedge \bigvee_{R}(R \in \Re(\Re) \wedge \mu(R)<\infty \wedge B \subset A \cap R)\right\}
$$

bedingtes $\sigma$-Ideal summierbarer Mengen im Sinne von Segal. Da mit dem Satz von Radon-Nikodym auch das Segal'sche Lokalisationsprinzip gilt, wird $\mathfrak{i}$ durch ein $L \in \mathfrak{B}_{\mu}^{*}(\mathfrak{I})$ lokalisiert. Sei $M \in \mathfrak{B}_{\mu}^{*}(\mathfrak{I})$ und $\mu(M)<\infty$. Aus $K \in \Re$ und $K \subset M \cap(A \triangle L)$ folgt $\mu(K)=0$ : Denn zu $K \cap L$ existiert ein $I \in \mathfrak{i}$ mit $\mu(K \cap L-I)=0$, da $L \mathfrak{i}$ lokalisiert. Wegen $I \subset A$ folgt $\bar{\mu}(K \cap L-A)=0$ und $\bar{\mu}(K \cap L)=\bar{\mu}(K \cap L \cap A)=\bar{\mu}(\emptyset)=0$. Es bleibt $K \cap A$ zu betrachten: Wegen $K \in \Re$ und $A \in \mathfrak{B}_{\mu}(\Re)$ gilt $K \cap A \in \Re_{\mu}(\Re)$, der 
Vervollständigung von $\Re(\Re)$. Es folgt $K \cap A \in \dot{i}$ und damit $\mu(K \cap A-L)$ =0. Damit erhält man $\bar{\mu}(K \cap A)=\mu(K \cap A \cap L)=0$ und insgesamt $\bar{\mu}(K) \leqslant \bar{\mu}(K \cap A)+\bar{\mu}(K \cap L)=0$. Damit gilt aber auch $\bar{\mu}(M \cap A \triangle L)$ $=0$ für alle $M \in \mathfrak{B}_{\mu}^{*}(\mathfrak{T})$ mit $\mu(M)<\infty . A \triangle L$ ist lokale Nullmenge und es folgt $A \in \mathfrak{B}_{\mu}^{*}(\mathfrak{T})$.

\section{LITERATUR}

[1] N. Bourbaki, Eléments de mathématique. Intégration Livre XIII, Chap. IV, Hermann, Paris.

[2] P. Courrège, Théorie de la mesure. Centre de Documentation Universtiaire, Paris (1962).

[ 3 ] W. T. van Est and H. Freudenthal, Trennung durch stetige Funktionen in topologischen Räumen. Indagationes Math. 13 (1951), 359-368.

[4] D. Kölzow, Differentiation von Maßen, Lecture Notes in Mathematics 65, Springer, Berlin (1968).

[ 5 ] I. E. Segal, Equivalence of measure spaces, Amer. J. Math. 73 (1951), 275-313.

[6] M. H. Stones, The generalized Weierstrass approximation theorem, Math. Mag. $21(1947 / 48), 237-254$.

[ 7 ] M. L. Wage, A generalization of Lusin's theorem, Proc. Amer. Math. Soc. 52 (1975), 327-332.

Universität Braunschweig 